\title{
CORRECTION
}

\section{Correction to: Microsatellite records for volume 10, issue 2}

Dan Yu ${ }^{1,2} \cdot$ Xueyun Ding $^{3} \cdot$ Zhi Zhang $^{2} \cdot$ Yu Zeng $^{1} \cdot$ Huanzhang Liu ${ }^{2} \cdot$ Lin Wang $^{4,5} \cdot$ Ye Gong $^{6} \cdot$ Xingtu Liu $^{4}$. Xianguo Lyu ${ }^{4}$ - Gregory R. McCracken ${ }^{7}$. Francisca V. Valenzuela-Aguayo ${ }^{8}$. Evelyn M. Habit ${ }^{8}$. Daniel E. Ruzzante ${ }^{7}$. Min $\mathrm{Du}^{9} \cdot$ Wentao Wang ${ }^{9} \cdot$ Taowen Song $^{9} \cdot$ Baozhen Niu ${ }^{9} \cdot$ Qilin Zhang $^{9} \cdot$ Catherine L. Simmons ${ }^{10,11}$. Tamara K. Taylor ${ }^{12}$. Jacinta M. Zalucki ${ }^{12}$. Susan E. Hoebee ${ }^{13}$. Denise R. Fernando ${ }^{13}$. Gareth D. Holmes ${ }^{13}$. Alison Shapcott ${ }^{10} \cdot$ Zhiyong Zhang $^{14} \cdot$ Xiaolan Yan $^{15} \cdot$ Qixiang Zhang ${ }^{14}$

Published online: 24 July 2018

(c) Springer Nature B.V. 2018

\section{Correction to:}

Conservation Genetics Resources (2018) 10:269-276

https://doi.org/10.1007/s12686-018-1046-8

The author group of the article is not displayed in the web/ online version, though the information appeared in print version. The missing author group is provided in this Correction.

The original article can be found online at https://doi.org/10.1007/ s12686-018-1046-8.

\section{Dan Yu}

yudan@ihb.ac.cn

Extended author information available on the last page of the article 


\section{Affiliations}

\section{Dan Yu ${ }^{1,2} \cdot$ Xueyun Ding ${ }^{3} \cdot$ Zhi Zhang $^{2} \cdot$ Yu Zeng ${ }^{1} \cdot$ Huanzhang Liu ${ }^{2} \cdot$ Lin Wang $^{4,5} \cdot$ Ye Gong $^{6} \cdot$ Xingtu Liu $^{4}$. Xianguo Lyu ${ }^{4}$ - Gregory R. McCracken ${ }^{7}$. Francisca V. Valenzuela-Aguayo ${ }^{8}$ - Evelyn M. Habit ${ }^{8}$. Daniel E. Ruzzante ${ }^{7}$. Min $\mathrm{Du}^{9} \cdot$ Wentao Wang ${ }^{9} \cdot$ Taowen Song $^{9} \cdot$ Baozhen Niu ${ }^{9} \cdot$ Qilin Zhang $^{9} \cdot$ Catherine L. Simmons ${ }^{10,11}$. Tamara K. Taylor $^{12}$. Jacinta M. Zalucki ${ }^{12}$. Susan E. Hoebee ${ }^{13}$. Denise R. Fernando ${ }^{13}$. Gareth D. Holmes ${ }^{13}$. Alison Shapcott ${ }^{10} \cdot$ Zhiyong Zhang $^{14} \cdot$ Xiaolan Yan $^{15} \cdot$ Qixiang Zhang ${ }^{14}$}

Huanzhang Liu

hzliu@ihb.ac.cn

$\triangle$ Xianguo Lyu

luxg@neigae.ac.cn

$\triangle$ Gregory R. McCracken

gregory.mccracken@dal.ca

$\triangle$ Min Du

du2005min@126.com

$\triangle$ Catherine L. Simmons

Catherine.Laura.Simmons@gmail.com

Qixiang Zhang

zqxbjfu@126.com

1 Key Laboratory of Southwest China Wildlife Resources Conservation (Ministry of Education), College of Life Science, China West Normal University, Nanchong 637002, People's Republic of China

2 The Key Laboratory of Aquatic Biodiversity and Conservation of Chinese Academy of Sciences, Institute of Hydrobiology, Chinese Academy of Sciences, Wuhan 430072, People's Republic of China

3 Wenzhou Medical University, Wenzhou 325035, People's Republic of China

4 Key Laboratory of Wetland Ecology and Environment, Northeast Institute of Geography and Agroecology, Chinese Academy of Sciences, 4888 Shengbei Street, Changchun 130102, People's Republic of China

5 University of Chinese Academy of Sciences, Beijing 100049, People's Republic of China

6 School of Life Sciences, Jilin Key Laboratory of Animal Resource Conservation and Utilization, Northeast Normal University, 5268 Renmin Street, Changchun, People's Republic of China
7 Department of Biology, Dalhousie University, 1355 Oxford Street, Halifax, NS B3H 4R2, Canada

8 Departmento de Sistemas Acuáticos, Facultad de Ciencias Ambientales y Centro EULA, Universidad de Concepción, Casilla 160-C, Concepción, Chile

9 Key Laboratory of Crops with High Quality and Efficient Cultivation and Security Control, Yunnan Higher Education Institutions, College of Life Science and Technology, Honghe University, 661199 Mengzi, Yunnan Province, People's Republic of China

10 Genecology Research Centre, Faculty of Science Health, Education and Engineering, University of the Sunshine Coast, Maroochydore DC 4558, QLD, Australia

11 Queensland Herbarium, Department of Science, Information Technology and Innovation, Toowong 4066, QLD, Australia

12 Environmental Futures Research Institute, Griffith University, Nathan 4111, QLD, Australia

13 Department of Ecology, Environment and Evolution, La Trobe University, Bundoora 3086, VIC, Australia

14 Beijing Key Laboratory of Ornamental Plants Germplasm Innovation \& Molecular Breeding, National Engineering Research Center for Floriculture, Beijing Laboratory of Urban and Rural Ecological Environment, Key Laboratory of Genetics and Breeding in Forest Trees and Ornamental Plants of Ministry of Education, School of Landscape Architecture, Beijing Forestry University, Beijing 100083, People's Republic of China

15 Mei Flower Research Center in China, Wuhan 430074, People's Republic of China 